\title{
Xerostomia: a common adverse effect of drugs and radiation
}

\author{
Ian N Olver, Chief Executive Officer, The Cancer Council Australia, Sydney
}

\section{Summary}

Xerostomia is the subjective sensation of dry mouth. Many drugs, especially those with anticholinergic effects, can cause xerostomia, particularly in the elderly. Another major cause is radiotherapy to the head and neck damaging the salivary glands. Newer techniques to target radiotherapy and protective drugs, such as amifostine, could decrease the incidence of xerostomia. Treatment is based on either stimulating the flow of remaining salivary tissue with lollies or sialogogues such as pilocarpine, or wetting the mucosa with water or artificial saliva containing glycerine or compounds such as carboxymethylcellulose. Patients need regular dental examinations because of the effect of xerostomia on intra-oral health.

Key words: pilocarpine, radiotherapy, salivary glands, sialogogues.

(Aust Prescr 2006;29:97-8)

\section{Introduction}

Xerostomia is the subjective feeling of having a dry mouth. It is associated with dysfunction of the salivary glands. These glands normally produce 1-1.5 L of saliva each day. Saliva has a fluid component (responsible for lubrication) released by parasympathetic stimulation and a protein component released from secretory vesicles by sympathetic stimulation. Not only is lubrication a necessary part of chewing food prior to swallowing, but saliva impacts on the oral microbial environment, the maintenance of the oral immune system and also the mineralisation of teeth.

\section{Causes}

A variety of drugs can cause xerostomia. They include diuretics, beta blockers, tricyclic antidepressants, antihistamines, anticonvulsants and antipsychotics. Xerostomia is also reported with oral morphine. There is a greater likelihood of taking these drugs as patients age which explains the correlation of xerostomia with age.

Diseases such as Sjogren's syndrome with sicca symptoms, or endocrine conditions such as hypothyroidism can result in xerostomia. It is important to also consider psychological factors. Both anxiety and depression have been associated with decreased salivary flow rates.

Radiotherapy to the head and neck is a major cause of xerostomia. The prevalence of xerostomia post-radiation is over $90 \%$. It was found to be severe in $30 \%$ of patients with advanced cancer starting a palliative care program. ${ }^{1}$ Often patients with cancer become dehydrated, which will exacerbate the symptom of dry mouth.

Nerve damage during head and neck surgery may both compromise the function of the salivary glands and alter oral sensation. Chemotherapy can also decrease salivary flow.

The secretory cells of the parotid gland are very radiosensitive and radiation causes inflammation and vascular damage in the parenchyma. A decrease in salivary flow can occur within the first week of radiation, with the saliva becoming more viscous and acidic. There are also changes in the electrolyte and protein content of the saliva which in turn affects the microbial flora and the propensity for tooth decay. The degree of damage depends on how many of the major glands are in the radiation field and the damage increases with total dose of radiation. ${ }^{2}$ The initial volume, particularly of the parotid glands, will also affect the degree of xerostomia post-radiation. If only one side is irradiated some recovery will occur over the following year as a result of compensatory hypertrophy of the remaining glands.

\section{Symptoms}

The most frequent symptoms of xerostomia are the need to keep the mouth moist with water, particularly at night, and difficulty with speech. There is also a loss of taste and difficulty chewing and swallowing dry foods. This can in turn cause loss of appetite and weight. Patients find it difficult to tolerate dental prostheses and are more prone to dental caries. Burning and tingling sensations on the tongue are reported. Fissuring of the lips and mouth ulcers increase the chance of secondary fungal infections in the mouth.

\section{Evaluation}

History and examination, including palpation of the salivary glands, are most important. On inspection the buccal mucosa may be dry and sticky, or red if there is a secondary infection with Candida albicans. There is no pooled saliva. Lipstick adhering to the front teeth can be a sign of xerostomia as there is no saliva to clean their surfaces.

Sialometry is a specialist test which can be used to aid in the diagnosis of Sjogren's syndrome. Saliva is collected from the salivary gland orifices and the response to stimulation with citric acid can be measured, although this test is complex and can be inaccurate. The glands may be imaged with sialography or by 
nuclear medicine techniques. Salivary gland biopsy can be used to diagnose conditions such as Sjogren's syndrome.

\section{Prevention}

General nutrition and hydration are important to help reduce the severity of xerostomia. Avoiding or decreasing the doses of the many drugs which are associated with xerostomia, particularly in the elderly, is a useful precaution.

Patients are encouraged to regularly practice oral and dental hygiene, and if they wear dentures, not to leave them in at night. They should avoid sugary or acidic foods or irritating spicy foods. ${ }^{3}$ Stopping alcohol and smoking will help to decrease dental disease.

\section{Pre-radiation}

Before radiotherapy, patients should be reviewed by a dentist and have any necessary dental treatment, for example the application of fluoride gels. Antifungal drugs may be used to prevent candidiasis in patients at high risk of infection.

Another major strategy to try to prevent xerostomia is to better target the radiotherapy. Newer techniques including threedimensional planning and intensity-modulated radiation, where the dosage can be varied across the treatment volume, aim to spare the normal tissues including the salivary glands. In very specific cases, such as a head and neck cancer with no neck nodes involved where only one side of the neck is being irradiated, a submandibular gland can be surgically transferred to a shielded area in the submental space to protect it from the radiation.

Drugs are being developed to try to protect normal tissues from chemotherapy or radiation damage. Amifostine, a thiol free radical scavenger, has been shown to reduce the damage to salivary glands when given concomitantly with radiation such as radioiodine for treating the thyroid gland. The adverse effects of amifostine include nausea and hypotension and it needs to be injected daily. ${ }^{4}$

\section{Treatment}

The initial strategy to treat xerostomia is to try to stimulate any remaining salivary gland tissue. This may be achieved by chewing gum or by sucking sugarless lollies.

Sialogogues are drugs which stimulate saliva production. They are essentially muscarinic-cholinergic analogues which act on smooth muscle and exocrine glands. Pilocarpine can increase salivary flow after radiotherapy, but is associated with adverse effects such as perspiration, flushing and increased bowel and bladder motility. ${ }^{5}$ Its peak effects are within one hour of administration. Sialogogues are contraindicated in patients with asthma, congestive heart disease and narrow angle glaucoma. Recent studies of pilocarpine do not show that it improves salivary flow or quality of life. ${ }^{3}$ Cevimeline is more selective of $\mathrm{M}_{1}$ and $\mathrm{M}_{3}$ receptors and thus may minimise cardiac and pulmonary adverse effects. However, clinical trials will be needed to confirm its preclinical activity in radiation-induced xerostomia. ${ }^{6}$ Another drug used to stimulate salivary flow is interferon alfa lozenges as trialled in Sjogren's syndrome. The literature is equivocal on whether acupuncture has a role in alleviating xerostomia.

Some of the discomfort of xerostomia may be relieved by wetting the oral mucosa. This may be simply achieved by regular sips of water, but other substitutes for saliva contain mucin, glycerine or carboxymethylcellulose, hydroxypropylcellulose or hydroxyethylcellulose. These artificial salivas mimic the physical characteristics of saliva but do not have any of its antimicrobial properties. Trials have been conducted of intra-oral reservoirs containing artificial saliva to try to simulate salivary flow. ${ }^{7}$

\section{Conclusion}

Xerostomia is a common adverse effect associated with drugs and cancer therapy, particularly radiation to the head and neck. Appropriate drug therapy, good oral hygiene and better targeting of radiation may help prevent the disease. Strategies to alleviate symptoms include stimulating the remaining glands or using substitutes for saliva.

\section{References}

1. Ventafridda V, De Conno F, Ripamonti C, Gamba A, Tamburini M. Quality-of-life assessment during a palliative care program. Ann Oncol 1990;1:415-20.

2. Blanco Al, Chao KS, El Naqa I, Franklin GE, Zakarian K, Vicic $M$, et al. Dose-volume modeling of salivary function in patients with head-and-neck cancer receiving radiotherapy. Int J Radiat Oncol Biol Phys 2005;62:1055-69.

3. Gornitsky M, Shenouda G, Saltanem K, Katz H, Hier M, Black $\mathrm{M}$, et al. Double-blind randomized, placebo-controlled study of pilocarpine to salvage salivary gland function during radiotherapy of patients with head and neck cancer. Oral Surg Oral Med Oral Pathol Oral Radiol Endod 2004;98:45-52.

4. Sasse AD, Clark LG, Sasse EC, Clark OA. Amifostine reduces side effects and improves complete response rate during radiotherapy: results of a meta-analysis. Int J Radiat Oncol Biol Phys 2006;64:784-91.

5. Greenspan D, Daniels TE. Effectiveness of pilocarpine in postradiation xerostomia. Cancer 1987;59:1123-5.

6. Asari T, KomatsuY, Misawa K, Hara K, Akahane M. Prophylactic effects of pilocarpine hydrochloride on xerostomia models induced by $\mathrm{X}$-ray irradiation in rats. Clin Exp Pharmacol Physiol 2001;28:545-50.

7. VergoTJ Jr, Kadish SP. Dentures as artificial saliva reservoirs in the irradiated edentulous cancer patient with xerostomia: a pilot study. Oral Surg Oral Med Oral Pathol 1981;51:229-33.

\section{Further reading}

Ship JA, Pillemer SR, Baum BJ. Xerostomia and the geriatric patient. J Am Geriatr Soc 2002;50:535-43.

Conflict of interest: none declared

See Dental notes page 108 